\title{
Joint String Complexity for Markov Sources
}

\author{
Philippe Jacquet $^{1}$ and Wojciech Szpankowski ${ }^{2}$ \\ ${ }^{1}$ Bell Labs, Alcatel-Lucent, France. \\ ${ }^{2}$ Department of Computer Science, Purdue University, USA
}

\begin{abstract}
String complexity is defined as the cardinality of a set of all distinct words (factors) of a given string. For two strings, we define joint string complexity as the set of words that are common to both strings. We also relax this definition and introduce joint semi-complexity restricted to the common words appearing at least twice in both strings. String complexity finds a number of applications from capturing the richness of a language to finding similarities between two genome sequences. In this paper we analyze joint complexity and joint semi-complexity when both strings are generated by a Markov source. The problem turns out to be quite challenging requiring subtle singularity analysis and saddle point method over infinity many saddle points leading to novel oscillatory phenomena with single and double periodicities.
\end{abstract}

Keywords: String complexity, semi-complexity, double Mellin transform, double depoissonization, saddle point method.

\section{DEDICATED TO OUR FRIEND AND MENTOR PHILIPPE FLAJOLET}

\section{Introduction}

In the last decades, several attempts have been made to capture mathematically the concept of "complexity" of a sequence. The notion is connected with quite deep mathematical properties, including the rather elusive concept of randomness in a string (see e.g., [3, 10, 11]).

We are interested in studying some measures of complexity of a set of strings. We recall that the string (sequence) complexity for a single string is defined as the cardinality of the set of distinct words (factors) of a given string (that is, words that occur exactly once in the string) [9]. For two strings the joint complexity is the cardinality of the set of common words to both strings, while the joint semi-complexity is the set of words that occur at least twice in both strings. Hereafter, we mostly analyze the average joint semicomplexity when both strings are generated by non-identical Markov sources. In fact, in this conference version, to avoid cumbersome notation and much longer derivations, we only present (asymptotics) results when one of the source is uniform.

String complexity has a number of applications. It captures the "richness of the language" used in a sequence. For example, sequences with low complexity contain a large number of repeated substrings and

\footnotetext{
${ }^{\dagger}$ This work was supported in part by the NSF Science and Technology Center for Science of Information Grant CCF-0939370, NSF Grants CCF-0830140 and DMS-0800568, AFOSR Grant FA8655-11-1-3076, NSA Grant H98230-11-1-0141, and the MNSW Grant N206 369739.
}

1365-8050 @ 2012 Discrete Mathematics and Theoretical Computer Science (DMTCS), Nancy, France 
they eventually become periodic (e.g., tandem repeats in a DNA sequence). In order to identify unusually low- or high-complexity strings one needs to determine how the complexities of the strings under study deviate from the average or maximum string complexity. On the other hand, joint string complexity is an efficient way of evaluating similarity degree of two sequences. For example, genome sequences of two dogs will contain more common words than genome sequences of a dog and a cat. Similarly, the set of common words of one author's texts is larger than the set of common words between two texts from two different authors. Finally, as our analysis shows the joint complexity or the joint semi-complexity can be used to discriminate between identically distributed sources and non-identical sources. Indeed, we shall prove that for non identical sources, the string joint complexity is of order $O\left(n^{\kappa}\right)$ for some $\kappa<1$ when both strings are of length $n$. When the sources are identically distributed the complexity is $O(n)$ (not treated in this paper). Furthermore, if both strings are identical (i.e. we deal with a single string complexity), then the string complexity is $O\left(n^{2}\right)[9]$.

Single string complexity was studied extensively in the past. The literature is reviewed in [9] where precise analysis of string complexity is discussed for strings generated by unbiased memoryless sources. Another analysis of the same situation was also proposed in [4] where for the first time the joint string complexity for memoryless sources is presented. It was evident from [4] that precise analysis of the joint complexity is quite challenging due to intricate singularity analysis and infinite number of saddle points. In this paper we deal with the joint string complexity and semi-complexity for Markov sources. To the best of our knowledge this problem was never tackled before. As expected, its analysis is very sophisticated but at the same time quite rewarding. It requires not only generalized (two-dimensional) depoissonization and generalized (two-dimensional) Mellin transforms but also subtle singularity analysis and unusual saddle point over infinity many saddle points. Furthermore, unlike other similar analyses [12] the non-binary case leads to new oscillatory phenomenon with single and double periodicities. In the long version of this paper, we shall also show that traditional dichotomy between rational and irrational cases must be extended to commensurability and non-commensurability.

\section{Main Results}

In this section we first define precisely the joint string complexity and semi-complexity. Hereafter, we mostly concentrate on the semi-complexity. Then we derive the basic functional equation describing the average joint semi-complexity for two strings generated by Markov sources. Finally, in this conference version we present asymptotic results only for a simplified version when one of the string is generated by an unbiased source while the other source is Markovian.

We begin by introducing some general notation. Let $\omega$ and $\sigma$ be two strings over alphabet $\mathcal{A}$. We denote by $|\omega|_{\sigma}$ the number of times $\sigma$ occurs in $\omega$. For example, $|a b b b a|_{b b}=2$. By convention $|\omega|_{\epsilon}=|\omega|$, where $\epsilon$ is an empty string.

Throughout we denote by $X$ a string (text) whose complexity we plan to study. We also assume that its length $|X|$ is equal to $n$. We study the string semi-complexity of a single string $X$, that is, the set of distinct substrings of $X$ that occur twice in $X$, excluding the empty string $\epsilon$. We denote this set as $S_{X}$ : $S_{X}:=\left\{\omega: \omega \neq \epsilon \&|X|_{\omega} \geq 2\right\}$. For example, if $X=a a b a a$, then $S_{X}=\{a, b, a a\}$. We also have

$$
\left|S_{X}\right|=\sum_{\sigma \in \mathcal{A}^{*}} 1_{|X|_{\sigma}>1}
$$

where $1_{A}$ is the indicator function of $A$. It is also related to the number of nodes in the associated suffix 
tree of $X$ [13] (see also [5]). In passing we point out that the average string complexity for memoryless sources was studied in Janson at al. [9] and Jacquet [4].

Now, let $X$ and $Y$ be two sequences (not necessarily of the same length). We define the joint semicomplexity as the cardinality of the set $S_{X, Y}=S_{X} \cap S_{Y}$ of common words that appear at least twice in both strings, excluding the empty string. For example, if $X=a a b a a$ ad $Y=a b b b a$, then $S_{X, Y}=\{a\}$. In fact, the joint semi-complexity corresponds to the number of common nodes in two suffix trees built from $X$ and $Y$ (excluding the root), and

$$
\left|S_{X, Y}\right|=\sum_{\sigma \in \mathcal{A}^{*}} 1_{|X|_{\sigma}>1} \times 1_{|Y|_{\sigma}>1} .
$$

In this conference paper, we only analyze the average joint semi-complexity. Extension to the joint complexity is rather straightforward and it is left for the full paper. We now assume that both strings $X$ and $Y$ are generated by two independent Markov sources with the transition probabilities $P_{i}(a \mid b)$ for source $i \in\{1,2\}$, where $(a, b) \in \mathcal{A}^{2}$. We denote by $\mathbf{P}_{1}$ (resp. $\mathbf{P}_{2}$ ) the transition matrix of Markov source 1 (resp. source 2). The stationary distributions are denoted by $\pi_{1}(a)$ and $\pi_{2}(a)$ for $a \in \mathcal{A}$, respectively.

We denote by $S_{n, m}$ the average string semi-complexity, that is, the average number of common words occurring at least twice in two strings generated by stationary Markov sources. As in [5], we can prove that

$$
S_{n, m}=T_{n, m}+O\left(n^{-\epsilon}\right),
$$

where $T_{m, n}$ is the average number of common internal nodes between two tries $T_{1}$ and $T_{2}$ built over $n$ and $m$ independent strings, respectively, generated by Markov sources. We should point out that a single trie built over a Markov source was already analyzed in [8].

Let $a \in \mathcal{A}$. We denote $T_{a, m, n}$ the average number of common internal nodes between two tries when all $n+m$ independent strings start with symbol $a$. Notice that $T_{a, m, n}=0$ for $n, m \leq 1$. Furthermore $T_{a, n, m}$ for $n, m \geq 2$ satisfies the following recurrence for all $b \in \mathcal{A}$

$$
T_{b, n, m}=1+\sum_{a \in \mathcal{A}} \sum_{n_{a}, m_{a}}\left(\begin{array}{c}
n \\
n_{a}
\end{array}\right)\left(\begin{array}{c}
m \\
m_{a}
\end{array}\right)\left(P_{1}(a \mid b)\right)^{n_{a}}\left(1-P_{1}(a \mid b)\right)^{n-n_{a}}\left(P_{2}(a \mid b)\right)^{m_{a}}\left(1-P_{2}(a \mid b)\right)^{m-m_{a}} T_{a, n_{a}, m_{a}}
$$

where $n_{a}$ and $m_{a}$ denote, respectively, the number of " $a$ " occurrences in the first and the second string. Moreover, the unconditional average $T_{n, m}$ satisfies for $n, m \geq 2$

$$
T_{n, m}=1+\sum_{a \in \mathcal{A}} \sum_{n_{a}, m_{a}}\left(\begin{array}{c}
n \\
n_{a}
\end{array}\right)\left(\begin{array}{c}
m \\
m_{a}
\end{array}\right) \pi_{1}^{n_{a}}(a)\left(1-\pi_{1}(a)\right)^{n-n_{a}} \pi_{2}^{m_{a}}(a)\left(1-\pi_{2}(a)\right)^{m-m_{a}} T_{a, n, m} .
$$

The above recurrence follows from the fact that every strings in tries $T_{1}$ and $T_{2}$ have their first symbol distributed according to the stationary distribution. The double Poisson transform of $T_{a, n, m}$

$$
T_{a}\left(z_{1}, z_{2}\right)=\sum_{n, m \geq 0} T_{a, n, m} \frac{z_{1}^{n} z_{2}^{m}}{n ! m !} e^{-z_{1}-z_{2}}
$$

translates the above recurrence into the following functional equation:

$$
T_{b}\left(z_{1}, z_{2}\right)=\left(1-\left(1+z_{1}\right) e^{-z_{1}}\right)\left(1-\left(1+z_{2}\right) e^{-z_{2}}\right)+\sum_{a \in \mathcal{A}} T_{a}\left(P_{1}(a \mid b) z_{1}, P_{2}(a \mid b) z_{2}\right) .
$$


Furthermore, the cumulative double Poisson transform

$$
T\left(z_{1}, z_{2}\right)=\sum_{n, m \geq 0} T_{n, m} \frac{z_{1}^{n} z_{2}^{m}}{n ! m !} e^{-z_{1}-z_{2}}
$$

of the unconditional average joint semi-complexity $T_{n, m}$ satisfies

$$
T\left(z_{1}, z_{2}\right)=\left(1-\left(1+z_{1}\right) e^{-z_{1}}\right)\left(1-\left(1+z_{2}\right) e^{-z_{2}}\right)+\sum_{a \in \mathcal{A}} T_{a}\left(\pi_{1}(a) z_{1}, \pi_{2}(a) z_{2}\right) .
$$

In the next section we shall first analyze $T\left(z_{1}, z_{2}\right)$ for large $z_{1}, z_{2} \rightarrow \infty$ in a cone around the real axes, and then recall two-dimensional (double) analytic depoissonization (cf. Section 3.4) to translate $T\left(z_{1}, z_{2}\right.$ ) into $T_{n, m}$ by observing that $T_{n, m} \sim T(n, m)$. In order to analyze asymptotically (as $z_{1}, z_{2} \rightarrow \infty$ ) the above system of functional equations (3) \& (5), we resort to the double Mellin transform [1, 13]. We define the double Mellin transform of $T_{a}\left(z_{1}, z_{2}\right)$ as

$$
\tilde{T}_{a}\left(s_{1}, s_{2}\right)=\int_{0}^{\infty} \int_{o}^{\infty} z_{1}^{s_{1}-1} z_{2}^{s_{2}-1} T_{a}\left(z_{1}, z_{2}\right) d z_{1} d z_{2} .
$$

Notice that for any $a \in \mathcal{A}: T_{a}\left(z_{1}, z_{2}\right)=O\left(z_{1}^{2} z_{2}^{2}\right)$ when $z_{1}, z_{2} \rightarrow 0$ and $T_{a}\left(z_{1}, z_{2}\right)=O\left(\left|z_{1}\right|+\left|z_{2}\right|\right)$ when $z_{1}, z_{2} \rightarrow \infty$. Thus the Mellin transform is defined for $-2<\Re\left(s_{1}\right), \Re\left(s_{2}\right)<-1$.

Applying basic properties of the Mellin transform [1, 13] we find for all $b \in \mathcal{A}$

$$
\tilde{T}_{b}\left(s_{1}, s_{2}\right)=\left(s_{1}+1\right) \Gamma\left(s_{1}\right)\left(s_{2}+1\right) \Gamma\left(s_{2}\right)+\sum_{a \in \mathcal{A}}\left(P_{1}(a \mid b)\right)^{-s_{1}}\left(P_{2}(a \mid b)\right)^{-s_{2}} \tilde{T}_{a}\left(s_{1}, s_{2}\right) .
$$

Let now $\mathbf{T}\left(s_{1}, s_{2}\right)$ be the vector consisting of $\tilde{T}_{a}\left(s_{1}, s_{2}\right)$ for $a \in \mathcal{A}$. Then

$$
\mathbf{T}\left(s_{1}, s_{2}\right)=\left(s_{1}+1\right) \Gamma\left(s_{1}\right)\left(s_{2}+1\right) \Gamma\left(s_{2}\right) \mathbf{1}+\mathbf{P}\left(s_{1}, s_{2}\right) \mathbf{T}\left(s_{1}, s_{2}\right)
$$

where $\mathbf{1}$ is the unit vector composed of 1's, and $\mathbf{P}\left(s_{1}, s_{2}\right)$ is the matrix whose $(a, b)$ elements are $\left.P_{1}(a \mid b)\right)^{-s_{1}}\left(P_{2}(a \mid b)\right)^{-s_{2}}$. In other words, $\mathbf{P}\left(s_{1}, s_{2}\right)=\mathbf{P}_{1}^{-s_{1} *} * \mathbf{P}_{2}^{-s_{2} *}$, where $*$ indicates the Schur product (i.e. element-wise) and $\mathbf{P}^{x *}$ is the Schur power obtained by raising all elements of $\mathbf{P}$ to the power $x$.

Putting all together we arrive at the following matrix equation

$$
\mathbf{T}\left(s_{1}, s_{2}\right)=\left(s_{1}+1\right) \Gamma\left(s_{1}\right)\left(s_{2}+1\right) \Gamma\left(s_{2}\right)\left(\mathbf{I}-\mathbf{P}\left(s_{1}, s_{2}\right)\right)^{-1} \mathbf{1} .
$$

Moreover, if $\tilde{T}\left(s_{1}, s_{2}\right)$ is the double Mellin transform of $T\left(z_{1}, z_{2}\right)$, then from 5 , we find

$$
\tilde{T}\left(s_{1}, s_{2}\right)=\left(s_{1}+1\right) \Gamma\left(s_{1}\right)\left(s_{2}+1\right) \Gamma\left(s_{2}\right)\left(1+\left\langle\boldsymbol{\pi}\left(s_{1}, s_{2}\right) \mid\left(\mathbf{I}-\mathbf{P}\left(s_{1}, s_{2}\right)\right)^{-1} \mathbf{1}\right\rangle\right),
$$

where $\boldsymbol{\pi}\left(s_{1}, s_{2}\right)$ is the vector $\left[\left(\pi_{1}(a)\right)^{-s_{1}}\left(\pi_{2}(a)\right)^{-s_{2}}\right]_{a \in \mathcal{A}}$ and $\langle\cdot \mid \cdot\rangle$ denotes the scalar product.

As pointed out in the introduction, we focus in this conference paper on asymptotics of a simpler model in which one of the source is uniform and memoryless, that is, $P_{1}(a \mid b)=\frac{1}{|\mathcal{A}|}$, or $\mathbf{P}_{1}=\frac{1}{|\mathcal{A}|} \mathbf{1} \otimes \mathbf{1}$, and when $\mathbf{P}_{2} \neq \mathbf{P}_{1}$. To simplify our notation for all $(a, b) \in \mathcal{A}^{2}$ we shall write $P_{2}(a \mid b)=P(a \mid b)$ and $\mathbf{P}_{2}=\mathbf{P}$. Therefore

$$
\mathbf{P}\left(s_{1}, s_{2}\right)=|\mathcal{A}|^{s_{1}} \mathbf{P}(s)
$$


with $\mathbf{P}(s)=\mathbf{P}^{-s_{2} *}$. We also write $\pi(a)=\pi_{2}(a)$ and $\pi(s)=\left[\pi(a)^{-s}\right]_{a \in \mathcal{A}}$, thus

$$
\boldsymbol{\pi}\left(s_{1}, s_{2}\right)=|\mathcal{A}|^{s_{1}} \boldsymbol{\pi}\left(s_{2}\right) .
$$

Let $\lambda\left(s_{1}, s_{2}\right)$ be the main (largest) eigenvalue of $\mathbf{P}\left(s_{1}, s_{2}\right)$. We have

$$
\left.\lambda\left(s_{1}, s_{2}\right)\right)=|\mathcal{A}|^{s_{1}} \lambda\left(s_{2}\right)
$$

where $\lambda(s)$ is the main eigenvalue of matrix $\mathbf{P}(s)$. We also define $\mathbf{u}(s)$ as the right eigenvector of $\mathbf{P}^{-s *}$ and $\boldsymbol{\zeta}(s)$ as the left eigenvector provided $\langle\boldsymbol{\zeta}(s) \mid \mathbf{u}(s)\rangle=1$.

We now present a brief road map to an asymptotic analysis of $T_{n, n}$ as $n \rightarrow \infty$. We shall apply (double) depoissonization to $T(z, z)$ as $z \rightarrow \infty$ in a cone around the real axis. To recover $T(z, z)$ asymptotically, we use the inverse double Mellin transform. From (10) we find for all $-2<\rho<-1$ :

$$
T(z, z)=\frac{1}{(2 i \pi)^{2}} \iint_{\Re\left(s_{1}\right)=\Re\left(s_{2}\right)=\rho} \Xi\left(s_{1}\right) \Xi\left(s_{2}\right)\left(1+\left\langle\boldsymbol{\pi}\left(s_{1}, s_{2}\right) \mid\left(\mathbf{I}-\mathbf{P}\left(s_{1}, s_{2}\right)\right)^{-1} \mathbf{1}\right\rangle\right) z^{-s_{1}-s_{2}} d s_{1} d s_{2},
$$

where $\Xi(s):=(s+1) \Gamma(s)$. Let now $\left.\lambda_{1}(s), \lambda_{2}(s) \ldots, \lambda_{|\mathcal{A}|}(s)\right)$ be the eigenvalues of $\mathbf{P}(s)$ in the nonincreasing order of their modulus with $\lambda(s):=\lambda_{1}(s)$. To evaluate asymptotically the above integral, we move the line of integration with respect to $s_{1}$ from $\rho$ to $M$ for some $M>1$. We shall notice that $\left(\mathbf{I}-\mathbf{P}\left(s_{1}, s_{2}\right)\right)^{-1}$ ceases to exist at $|\mathcal{A}|^{s_{1}} \lambda_{i}\left(s_{2}\right)=1$. These simple poles satisfy

$$
s_{1}:=L_{i, k}\left(s_{2}\right)=-\frac{1}{\log |\mathcal{A}|}\left(\log \lambda_{i}\left(s_{2}\right)+2 i k \pi\right)
$$

for $i \in\{1, \ldots,|\mathcal{A}|\}$ and $k \in \mathbb{Z}$. The residues of these poles are exactly equal to

$$
I(z, \rho)=\frac{1}{2 i \pi} \int_{\Re(s)=\rho} \sum_{k \in \mathbb{Z}} \sum_{i=1}^{|\mathcal{A}|} \frac{f_{i}(s) g_{i}\left(s_{2}\right) \Xi\left(-L_{i, k}(s)\right) \Xi(s)}{\lambda_{i}(s) \log |\mathcal{A}|} z^{L_{i, k}(s)-s} d s .
$$

where $f_{i}(s)=\left\langle\boldsymbol{\pi}(s) \mid \mathbf{u}_{i}(s)\right\rangle$ and $g_{i}(s)=\left\langle\boldsymbol{\zeta}_{i}(s) \mid \mathbf{1}\right\rangle$. Furthermore, there is a pole at $s_{1}=0$ of $\Gamma\left(s_{1}\right)$ with residue 1 . Thus by the Cauchy residue theorem we find $T(z, z)=I(z, \rho)+1+O\left(z^{1-M}\right)$. The final task is to evaluate asymptotically $I(z, \rho)$. This depends on the growth of $z^{L(s)-s}$, where $L(s):=-L_{1,0}(s)=$ $\log _{|\mathcal{A}|} \lambda(s)$, and poles of the gamma function at $s=0$. The former is determined by the saddle point method with saddle points coinciding with the solution of $L^{\prime}(s)=1$. Detailed analysis is presented in the next section.

We now summarize our main results. First, we need some properties of $L(s)=\log _{|\mathcal{A}|} \lambda(s)$. In the Appendix we prove the following lemma.

Lemma 1 The function $L(s)$ is convex when s is real.

Let $c_{2}$ be the value that minimizes $L(s)-s$, that is,

$$
\frac{\lambda^{\prime}\left(c_{2}\right)}{\lambda\left(c_{2}\right)}=\log |\mathcal{A}| \text {. }
$$

Let also $c_{1}=-L\left(c_{2}\right)$ and $\kappa=-c_{1}-c_{2}=\min _{s}\{L(s)-s\}$. We have $\kappa \leq 1$ since $L(0)=1$. The next lemma is proved in the Appendix. 
Lemma 2 We always have $c_{2}>-1$. When all $(a, b) \in \mathcal{A}^{2}, P(a \mid b)>0$, then $c_{2}<0$.

We now can formulate our main result.

Theorem 1 Let $f(s)=\langle\boldsymbol{\pi}(s) \mid \mathbf{u}(s)\rangle$ and $g(s)=\langle\boldsymbol{\zeta}(s) \mid \mathbf{1}\rangle$. Furthermore, with $\Psi(s)$ being the Euler psi function, define $\alpha_{2}=L^{\prime \prime}\left(c_{2}\right)$ and

$$
\begin{aligned}
\beta_{2}\left(s_{1}, s_{2}\right) & =-\alpha_{2}\left(\Psi\left(s_{1}\right)+\frac{1}{1+s_{1}}+\log |\mathcal{A}|\right)+\Psi^{\prime}\left(s_{1}\right)-\frac{1}{\left(s_{1}+1\right)^{2}}+\Psi^{\prime}\left(s_{2}\right)-\frac{1}{\left(s_{2}+1\right)^{2}} \\
& +\frac{f^{\prime \prime}\left(s_{2}\right)}{f\left(s_{2}\right)}-\left(\frac{f^{\prime}\left(s_{2}\right)}{f\left(s_{2}\right)}\right)^{2}+\frac{g^{\prime \prime}\left(s_{2}\right)}{g\left(s_{2}\right)}-\left(\frac{g^{\prime}\left(s_{2}\right)}{g\left(s_{2}\right)}\right)^{2} .
\end{aligned}
$$

(i) If $c_{2}<0$ and $\kappa=-c_{1}-c_{2}$ with $c_{1}=-\log _{|\mathcal{A}|} \lambda\left(c_{2}\right)$, then

$$
T_{n, n}=n^{\kappa} \frac{f\left(c_{2}\right) g\left(c_{2}\right)\left(c_{1}+1\right) \Gamma\left(c_{1}\right)\left(c_{2}+1\right) \Gamma\left(c_{2}\right)}{\lambda\left(c_{2}\right) \log |\mathcal{A}| \sqrt{2 \pi\left(\alpha_{2} \log n+\beta_{2}\left(c_{1}, c_{2}\right)\right)}}+n^{\kappa} Q(\log n)+o\left(\frac{n^{\kappa}}{\sqrt{\log n}}\right),
$$

where depending on certain properties of $\mathbf{P}\left(c_{2}\right)$ the quantity $\sqrt{x} Q(x)$ tends to a periodic or a double periodic function when $x \rightarrow \infty$ (with amplitude of order $10^{-6}$ ), as detailed in Theorem 2.

(ii) If $c_{2}>0$, then

$$
T_{n, n}=\sum_{k \in \mathbb{Z}} \frac{f(0) g(0)}{\lambda(0)} \frac{\left(1-L(0)-\frac{2 i k \pi}{\log |\mathcal{A}|}\right) \Gamma\left(-L(0)-\frac{2 i k \pi}{\log |\mathcal{A}|}\right)}{\log |\mathcal{A}|} n^{L(0)+2 i k \pi / \log |\mathcal{A}|}+O\left(n^{L(0)-\epsilon}\right) .
$$

In order to present succinctly properties of the function $Q(x)$, we need to introduce more notations. We say that matrix $\mathbf{P}$ is rationally balanced if there exists $c \in \mathcal{A}$ and $\nu \in \mathbb{R}$ such that

$$
\nu(P(a \mid b)+P(c \mid a)-P(c \mid b)) \in \mathbb{Z} .
$$

Notice that this property does not depend on the pivot symbol $c$. In fact, we apply this to a matrix $\log ^{*}(\mathbf{M})$ whose elements are logarithm of the elements of some matrix $\mathbf{M}$.

Finally, let $\partial \mathcal{K}$ be the set of complex tuples $\left(s_{1}, s_{2}\right)$ satisfying $|\mathcal{A}|^{s_{1}} \lambda\left(s_{2}\right)=1$ such that $\Re\left(s_{1}\right)=c_{1}$ and $\Re\left(s_{2}\right)=c_{2}$. Notice that $\left(c_{1}, c_{2}\right) \in \partial \mathcal{K}$. We define $\partial \mathcal{K}^{*}=\partial \mathcal{K}-\left\{\left(c_{1}, c_{2}\right)\right\}$.

Theorem 2 The function $Q(x)$ introduced in Theorem 1 can be expressed as

$$
Q(x)=\sum_{\left(s_{1}, s_{2}\right) \in \partial \mathcal{K}^{*}} e^{i x \Im\left(s_{1}+s_{2}\right)} \frac{f\left(s_{2}\right) g\left(s_{2}\right)\left(s_{1}+1\right)\left(s_{2}+1\right) \Gamma\left(s_{1}\right) \Gamma\left(s_{2}\right)}{\lambda\left(s_{2}\right) \log |\mathcal{A}| \sqrt{2 \pi\left(\alpha_{2} x+\beta_{2}\left(s_{1}, s_{2}\right)\right)}} .
$$

If the matrix $\log ^{*}\left(\frac{1}{P(x \mid x)} \mathbf{P}\right)$ is rationally balanced (i.e., rational case), then let $\nu$ be the smallest non negative real such that

$$
\nu \log \left(\frac{1}{P(c \mid c)} \mathbf{P}\right) \in \mathbb{Z}^{|\mathcal{A}|^{2}} .
$$

Then

$$
\partial \mathcal{K}=\left\{\left(c_{1}+\frac{2 i k \pi}{\log |\mathcal{A}|}+2 i \pi \ell \nu \frac{\log P(c \mid c)}{\log |\mathcal{A}|}, c_{2}+2 i \pi \ell \nu\right),(k, \ell) \in \mathbb{Z}^{2}\right\},
$$


and $\sqrt{x} Q(x)$ is asymptotically double periodic. Otherwise (i.e., irrational case),

$$
\partial \mathcal{K}=\left\{\left(c_{1}+\frac{2 i k \pi}{\log 2}, c_{2}\right), k \in \mathbb{Z}\right\}
$$

and $\sqrt{x} Q(x)$ is asymptotically single periodic.
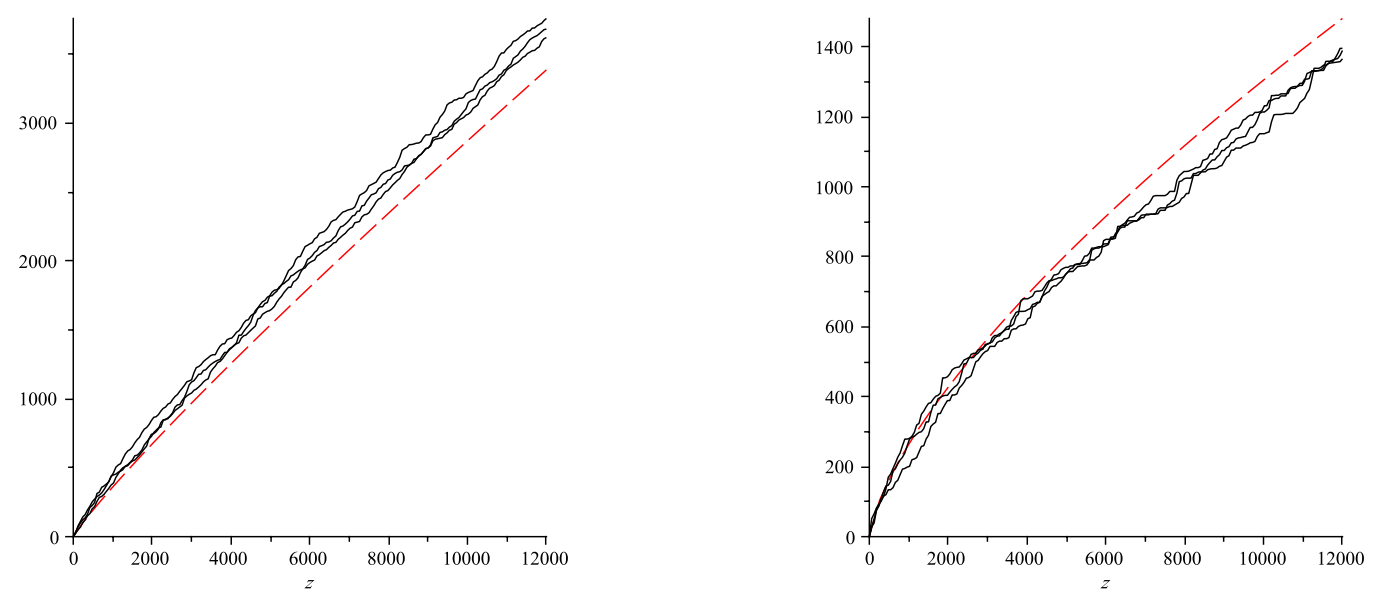

Fig. 1: Joint semi-complexity: three simulated trajectories (black) versus asymptotic average (dashed red); case $c_{2}>0$ (left) and case $c_{2}<0$ (right).

In Figure 1 we plot the joint semi-complexity for several pairs of strings $X$ and $Y$. String $X$ is generated by a Markov source with the transition matrix $\mathbf{P}$, and string $Y$ is generated by a uniform memoryless source. We consider two Markov sources for $X$ with the following transition matrix:

$$
\mathbf{P}=\left[\begin{array}{cc}
0 & 0.5 \\
1 & 0.5
\end{array}\right] \quad \mathbf{P}=\left[\begin{array}{cc}
0.2 & 0.8 \\
0.8 & 0.2
\end{array}\right]
$$

For the first $\mathbf{P}$ (left plot in Figure 1 ) we have $c_{2}>0$ (cf. Theorem[11ii)) while for the second $\mathbf{P}$ (right plot in Figure 1) we have $c_{2}<0$ (cf. Theorem 1 i i)).

\section{Analysis}

In this section we present the proof of our main result. We start with the double Mellin transform, followed by a brief discussion of properties of the set $\partial \mathcal{K}$ of the solutions of $|\mathcal{A}|^{s_{1}} \lambda\left(s_{2}\right)=1$. Then we turn our attention to the saddle point method and finally the double depoissonization. Some technical lemmas are postponed till the Appendix.

\subsection{Double Inverse Mellin Transform}

In 10 of previous section, we computed the double Mellin transform $\tilde{T}\left(s_{1}, s_{2}\right)$ of the double Poisson transform $T\left(z_{1}, z_{2}\right)$. We now recover $T\left(z_{1}, z_{2}\right)$ for large $z_{1}, z_{2} \rightarrow \infty$ around the real axes. In fact, we set 
$z_{1}=z_{2}=z$. Recall that by the inverse double Mellin transform [4] we have for all $-2<\rho<-1$ as in (14)

$$
\begin{aligned}
& T(z, z)=\frac{1}{(2 i \pi)^{2}} \iint_{\Re\left(s_{1}\right)=\Re\left(s_{2}\right)=\rho} \tilde{T}\left(s_{1}, s_{2}\right) z^{-s_{1}-s_{2}} d s_{1} d s_{2} \\
& =\frac{1}{(2 i \pi)^{2}} \iint_{\Re\left(s_{1}\right)=\Re\left(s_{2}\right)=\rho} \Xi\left(s_{1}\right) \Xi\left(s_{2}\right)\left(1+\left\langle\boldsymbol{\pi}\left(s_{1}, s_{2}\right) \mid\left(\mathbf{I}-\mathbf{P}\left(s_{1}, s_{2}\right)\right)^{-1} \mathbf{1}\right\rangle\right) z^{-s_{1}-s_{2}} d s_{1} d s_{2},
\end{aligned}
$$

where $\Xi(s):=(s+1) \Gamma(s)$. As before $\left.\lambda_{1}(s), \lambda_{2}(s) \ldots, \lambda_{|\mathcal{A}|}(s)\right)$ are the eigenvalues of $\mathbf{P}(s)$ in the non-increasing order (e.g., $\lambda(s):=\lambda_{1}(s)$ ) while $\mathbf{u}_{i}(s)$ and $\boldsymbol{\zeta}_{i}(s)$ ) are respectively the right and the left eigenvectors of $\mathbf{P}(s)$ associated with $\lambda_{i}(s)$ subject to $\left\langle\boldsymbol{\zeta}_{i}(s) \mid \mathbf{u}_{i}(s)\right\rangle=1$. By the spectral representation of matrices [13], we have

$$
\left(\mathbf{I}-\mathbf{P}\left(s_{1}, s_{2}\right)\right)^{-1}=\sum_{i=1}^{|\mathcal{A}|} \frac{1}{1-|\mathcal{A}|^{s_{1}} \lambda_{i}\left(s_{2}\right)} \mathbf{u}_{i}\left(s_{2}\right) \otimes \boldsymbol{\zeta}_{i}\left(s_{2}\right)
$$

where $\otimes$ denotes the tensor product. Observe that $\left(\mathbf{I}-\mathbf{P}\left(s_{1}, s_{2}\right)\right)^{-1}$ cease to exist at $\left(s_{1}, s_{2}\right)$ satisfying $|\mathcal{A}|^{s_{1}} \lambda_{i}\left(s_{2}\right)=1$, that is, for $s_{1}:=L_{i, k}\left(s_{2}\right)$ such that $L_{i, k}\left(s_{2}\right)=\frac{1}{-\log |\mathcal{A}|}\left(\log \lambda_{i}\left(s_{2}\right)+2 i k \pi\right)$, as already discussed in 15 .

The eigenvalues $\lambda_{i}(s)$ are individually analytic functions of $s$ as long as they are strictly decreasing (i.e. $\left|\lambda_{i-1}(s)\right|>\left|\lambda_{i}(s)\right|>\left|\lambda_{i+1}(s)\right|$ for all $i$ ). But any function of the form $\sum_{i} f\left(\lambda_{i}(s)\right)$ is analytic even when the eigenvalue sequence is not strictly decreasing, as long as $f()$ is analytic. To simplify our analysis, we also postulate that none of the eigenvalue is identically equal to zero, that is, we assume $\log \lambda_{i}(s)$ exists except on a countable set $\mathcal{R}=\left\{s: \exists i: \lambda_{i}(s)=0\right\}$. It should be pointed out that there are cases when some eigenvalues are identically equal to zero. For example, for memoryless sources we have for all $i \geq 2: \lambda_{i}(s) \equiv 0$. But these cases are easy to handle by just excluding these null eigenvalues; we will not address it here (see [4]).

Let $f_{i}(s)=\left\langle\boldsymbol{\pi}(s) \mid \mathbf{u}_{i}(s)\right\rangle$ and $g_{i}(s)=\left\langle\boldsymbol{\zeta}_{i}(s) \mid \mathbf{1}\right\rangle$. Define

$$
I(z, \rho)=\frac{1}{2 i \pi} \int_{\Re(s)=\rho} \sum_{k \in \mathbb{Z}} \sum_{i=1}^{|\mathcal{A}|} \frac{f_{i}(s) g_{i}\left(s_{2}\right) \Xi\left(-L_{i, k}(s)\right) \Xi(s)}{\lambda_{i}(s) \log |\mathcal{A}|} z^{L_{i, k}(s)-s} d s .
$$

Lemma 3 For any $M>0$ and $\rho \in]-2,-1[$, we have

$$
T(z, z)=I(z, \rho)+1+O\left(z^{1-M}\right) .
$$

Proof: As discussed in the previous section, to evaluate asymptotically 23, we move the line of integration with respect to $s_{1}$ from $\rho$ to $M$ for some $M>1$. There are simple poles at $L_{i, k}\left(s_{2}\right)$ for $i \in\{1, \ldots,|\mathcal{A}|\}$ and $k \in \mathbb{Z}$ with residues equal to $I(z, \rho)$ as in 25 . Furthermore, there is a pole at $s_{1}=0$ of $\Gamma\left(s_{1}\right)$ with residues 1 . Thus by the Cauchy residue theorem the estimate of $T(z, z)$ becomes

$$
T(z, z)=I(z, \rho)+\frac{1}{2 i \pi} \int_{\Re\left(s_{2}\right)=\rho} \Xi\left(s_{2}\right) z^{-s_{2}} d s_{2}+O\left(z^{1-M}\right) .
$$

But the above integral is equal to $1+O\left(z^{1-M}\right)$ (from the pole at $s_{2}=0$ ). 
Our task is to estimate asymptotically the integral $I(z, \rho)$ for $z \rightarrow \infty$ which depends on the growth of $z^{L(s)-s}$. We already know that $c_{2}$ defined in $(16)$ is the real saddle point determining the asymptotic behavior for $c_{2}<0$ (see Section 3.3 for details). But $\left.c_{2} \notin\right]-2,-1[$ and $I(z, \rho)$ may have additional singularities when $\rho$ moves towards $c_{2}$ that we must take this into account. When $c_{2}>0$ the important contribution is at $\rho=0$ due to the simple pole of function $\Xi(s)$. This case will be treated separately. Nevertheless, in the Appendix we prove the following lemma. The proof is rather technical since we have to cope with potential singularities between $\rho$ and $c_{2}$.

Lemma 4 If $c_{2}<0$, then for all $M>0$ we have $T(z, z)=I\left(z, c_{2}\right)+1+O\left(z^{1-M}\right)$.

\subsection{Properties of $\partial \mathcal{K}$ set}

Recall that $\partial \mathcal{K}$ is the set of complex tuples $\left(s_{1}, s_{2}\right)$ satisfying $|\mathcal{A}|^{s_{1}} \lambda\left(s_{2}\right)=1$ such that $\Re\left(s_{1}\right)=c_{1}$ and $\Re\left(s_{2}\right)=c_{2}$. Its structure is crucial for our asymptotic analysis, so we discuss it in this section. Among others, we show that if $\left(s_{1}, s_{2}\right) \in \partial \mathcal{K}$, then $\left(s_{1}+\frac{2 i k \pi}{\log |\mathcal{A}|}, s_{2}\right) \in \partial \mathcal{K}$ for all $k \in \mathbb{Z}$. We also show that the main eigenvalue of $\mathbf{P}$ is the dominant singularity. Throughout this section we assume that $c_{2}<0$.

Let us start with the structure of the set $\partial \mathcal{K}$. Let $\mathbf{P}$ be a matrix on $\mathcal{A} \times \mathcal{A}$ of complex coefficients $p_{a b}$ for all $(a, b) \in \mathcal{A}^{2}$. Let $\mathbf{Q}$ be a matrix $q_{a b}$. In the following we say $\mathbf{P}$ and $\mathbf{Q}$ are conjugate if there exists a non-zero complex vector $\left(x_{a}\right)_{a \in \mathcal{A}}$ such that $q_{a b}=\frac{x_{a}}{x_{b}} p_{a b}$. We say that such matrices are imaginary conjugate if $\left|x_{a}\right|=1$ for all $a \in \mathcal{A}$.

Observe that: (i) two conjugate matrices have the same eigenvalue set; (ii) if $\mathbf{u}=\left(u_{a}\right)_{a \in \mathcal{A}}$ is right eigenvector of $\mathbf{P}$, then $\left(x_{a} u_{a}\right)_{a \in \mathcal{A}}$ is right eigenvector of $\mathbf{Q}$. Similarly, if $\left(\zeta_{a}\right)_{a \in \mathcal{A}}$ is left eigenvector of $\mathbf{P}$, then $\left(\frac{1}{x_{a}} \zeta_{a}\right)_{a \in \mathcal{A}}$ is the left eigenvector of $\mathbf{Q}$.

The following lemma is essential and adapted from [8]. We prove it in the Appendix.

Lemma 5 Let $\mathbf{M}=\left[m_{a b}\right]_{(a, b) \in \mathcal{A}^{2}}$ be a matrix such that $m_{a b} \geq 0$. We assume that 1 is the largest eigenvalue of $\mathbf{M}$. Let $\mathbf{Q}$ be a matrix with coefficients $q_{a b}=e^{i \theta_{a b}} m_{a b}$ where $\theta_{a b}$ is real. The matrix $\mathbf{Q}$ has eigenvalue 1 if and only if $\mathbf{Q}$ is imaginary conjugate to matrix $\mathbf{M}$.

Corollary 1 Let $c \in \mathcal{A}$. The matrix $\mathbf{Q}$ has eigenvalue 1 if and only if for all $(a, b) \in \mathcal{A}^{2}$ :

$$
\frac{1}{2 \pi}\left(\theta_{a b}+\theta_{c a}-\theta_{c b}\right) \in \mathbb{Z} .
$$

Proof: We have $e^{i\left(\theta_{a}-\theta_{b}\right)}=\frac{e^{i \theta_{c b}}}{e^{i \theta_{c a}}}$, thus $e^{i\left(\theta_{c b}-\theta_{c a}\right)}=e^{i \theta_{a b}}$.

Lemma 6 Let $c \in \mathcal{A}$. A tuple $\left(s_{1}, s_{2}\right)$ belongs to $\partial \mathcal{K}$ iff for all $(a, b) \in \mathcal{A}^{2}$ we have

$$
\frac{\Im\left(s_{1}\right)}{2 \pi} \log |\mathcal{A}|-\frac{\Im\left(s_{2}\right)}{2 \pi} \log \frac{P(a \mid b) P(c \mid a)}{P(c \mid b)} \in \mathbb{Z} .
$$

Proof: Set $\mathbf{M}=\mathbf{P}\left(c_{1}, c_{2}\right)$ and $\mathbf{Q}=\mathbf{P}\left(s_{1}, s_{2}\right)$ for $\left(s_{1}, s_{2}\right) \in \partial \mathcal{K}$. Then, it follows directly from Corollary 1 with $e^{i \theta_{a b}}=|\mathcal{A}|^{i \Im\left(s_{1}\right)}(P(a \mid b))^{-i \Im\left(s_{2}\right)}$.

Now we focus on proving in Lemma 10 that the main eigenvalue is well separated.

Lemma 7 For all $s_{2}$ such that $\Re\left(s_{2}\right)=c_{2}$, we have $\nexists s_{1}:\left(s_{1}, s_{2}\right) \in \partial \mathcal{K} \Longleftrightarrow\left|\lambda\left(s_{2}\right)\right|<\lambda\left(c_{2}\right)$. 
Proof: By the Perron-Frobenius, we have $\left|\lambda\left(s_{2}\right)\right| \leq \lambda\left(c_{2}\right)$ since $\Re\left(s_{2}\right)=c_{2}$ and $\left|\mathbf{P}\left(s_{2}\right)\right|=\mathbf{P}\left(c_{2}\right)$ (by taking the modulus element-wise). If $\left|\lambda\left(s_{2}\right)\right|=\lambda\left(c_{2}\right)$, then there will be $t_{1}$ such that $|\mathcal{A}|^{i t_{1}} \lambda\left(s_{2}\right)=\lambda\left(c_{2}\right)$, and therefore $\left(c_{1}+i t_{1}, s_{2}\right) \in \partial \mathcal{K}$.

Lemma 8 We have $\lambda\left(c_{2}\right)>\lambda_{2}\left(c_{2}\right)$.

Proof: It follows from Perron-Frobenius that the main eigenvalue is unique.

Let $\mathcal{U}$ be a complex complex neighborhood of 0 such that $\forall s \in \mathcal{U}:\left|\lambda\left(c_{2}+s\right)\right|>\left|\lambda_{2}\left(c_{2}+s\right)\right|$. In the Appendix we prove the following lemma.

Lemma 9 Let $s_{k}$ be a sequence such that $\Re\left(s_{k}\right)=c_{2}$ and $\left|\lambda\left(s_{k}\right)\right| \rightarrow \lambda\left(c_{2}\right)$. Then for all $s \in \mathcal{U}$ we have

$$
\forall i: \lim _{k \rightarrow \infty} \frac{\lambda_{i}\left(s_{k}+s\right)}{\lambda\left(s_{k}+s\right)}=\frac{\lambda_{i}\left(c_{2}+s\right)}{\lambda\left(c_{2}+s\right)},
$$

and

$$
\begin{aligned}
\lim _{k \rightarrow \infty} L\left(s_{k}+s\right)-L\left(s_{k}\right) & =L\left(c_{2}+s\right)-L\left(c_{2}\right) \\
\lim _{k \rightarrow \infty} L^{\prime}\left(s_{k}+s\right) & =L^{\prime}\left(c_{2}+s\right) .
\end{aligned}
$$

where the convergence also holds for any further derivative of function $L^{\prime}(s)$.

In passing, we have $L^{\prime}\left(s_{k}\right) \rightarrow 1$ and $L^{\prime \prime}\left(s_{k}\right) \rightarrow \alpha_{2}$. Finally, we prove in the Appendix our second main lemma of this section.

Lemma 10 There exists $\epsilon>0$ such that for all $i \neq 1$ and for all s such that $\Re(s)=c_{2}$ :

$$
\left|\lambda_{i}(s)\right|<\lambda\left(c_{2}\right)-\epsilon .
$$

Proof: This is a consequence of previous lemmas. Suppose that there exists $s_{k}$ such that $\left|\lambda_{2}\left(s_{k}\right)\right| \rightarrow$ $\lambda\left(c_{2}\right)$. This implies that $\left|\lambda\left(s_{k}\right)\right| \rightarrow \lambda\left(c_{2}\right)$, but by previous lemma $\left|\lambda_{2}\left(s_{k}\right)\right| \rightarrow \lambda_{2}\left(c_{2}\right)=\lambda\left(c_{2}\right)-\epsilon$.

\subsection{Saddle Points Analysis}

We shall complete the proof by the saddle points analysis. We recall that for all $M>0: T(z, z)=$ $I\left(z, c_{2}\right)+1+O\left(z^{1-M}\right)$ where $I(z, c)$ is given in 25 . We assume $c_{2}<0$.

Lemma 11 There exists $\epsilon>0$ such that

$$
T(z, z)=\sum_{k \in \mathbb{Z}} \frac{1}{2 i \pi} \int_{\Re(s)=c_{2}} \frac{f_{1}(s) g_{1}(s) \Xi\left(-L_{1, k}(s)\right) \Xi(s)}{\lambda_{1}(s) \log |\mathcal{A}|} z^{L_{1, k}(s)-s} d s+O\left(z^{\kappa-\epsilon}\right)
$$

where $\kappa=-c_{1}-c_{2}$.

Proof: By Lemma 10 for all $i \neq 1$ we have $\log _{|\mathcal{A}|}\left|\lambda_{i}(s)\right|<\log _{|\mathcal{A}|} \lambda\left(c_{2}\right)-\epsilon$ for some $\epsilon>0$, thus other eigenvalues contribute negligibly. Let

$$
H_{i}(s, z)=\sum_{k \in \mathbb{Z}} \frac{f_{i}(s) g_{i}(s)}{\lambda_{i}(s) \log |\mathcal{A}|} \Xi\left(-L_{i, k}(s)\right) z^{L_{i, k}(s)} .
$$

The contribution of $\int_{\Re(s)=c_{2}} H_{i}(s, z) \Xi(s) z^{-s} d s$ is of order $\int_{\Re(s)=c_{2}}|\Xi(s)| z^{\Re\left(L_{i}(s)-s\right)} d s$ which in turn is of order $z^{L\left(c_{2}\right)-c_{2}-\epsilon}=z^{\kappa-\epsilon}$. 


\subsubsection{The Rational Case}

We assume now that the matrix $\log ^{*}\left(\frac{1}{P(c \mid c)} \mathbf{P}\right)$ is rationally balanced. The matrix $\mathbf{P}(s+2 i \pi \nu)$ is then imaginary conjugate with the matrix $P(c \mid c)^{2 i \pi \nu} \mathbf{P}(s)$ and $L(s+2 i \pi \nu)=L(s)+2 i \pi \nu \log P(c \mid c)$. Thus $\Re\left(L\left(c_{2}+i t\right)\right)$ is periodic in $t$ with period $2 \pi \nu$. Furthermore, $L^{\prime}(s)$ is also periodic with period $2 \pi \nu$. Thus, $s_{\ell}=c_{2}+2 i \pi \ell \nu$ for $\ell \in \mathbb{Z}$ are saddle points of $z^{L(s)-s}$.

We concentrate on the term $k=0$ of the right-hand side of 347. Define

$$
b_{2}(s)=\frac{d^{2}}{d s^{2}} \log \left(\frac{f(s) g(s)}{\lambda(s)} \Xi(-L(s)) \Xi(s)\right) .
$$

Notice that $b_{2}(s)=\beta_{2}(-L(s), s)$. Since the function

$$
\log \left(\frac{f(s) g(s)}{\lambda(s)} \Xi(-L(s)) \Xi(s)\right)
$$

has bounded variations, we have the classic saddle point result [2, 13]

$$
\begin{gathered}
\frac{1}{2 i \pi} \int_{\Re(s)=c_{2}} \frac{f(s) g(s)}{\lambda(s)} \Xi(-L(s)) \Xi(s) z^{L(s)-s} d s= \\
=\sum_{\ell} \frac{f\left(s_{\ell}\right) g\left(s_{\ell}\right)}{\lambda\left(s_{\ell}\right)} \Xi\left(-L\left(s_{\ell}\right)\right) \Xi\left(s_{\ell}\right) \frac{z^{L\left(s_{\ell}\right)-s_{\ell}}}{\sqrt{2 \pi\left(\alpha_{2} \log z+b_{2}\left(s_{\ell}\right)\right)}}(1+o(1)) .
\end{gathered}
$$

Notice that $\Re\left(L\left(s_{\ell}\right)-s_{\ell}\right)=\kappa$. When adding the contribution from the $L(s)+\frac{2 i k \pi}{\log |\mathcal{A}|}$ we obtain the expression for $Q(\log z)$ with $\partial \mathcal{K}=\left\{\left(-L\left(s_{\ell}\right)-\frac{2 i k \pi}{\log |\mathcal{A}|}, s_{\ell}\right),(k, \ell) \in \mathbb{Z}^{2}\right\}$. The double periodicity comes from the fact that $\sqrt{x} Q(x)=\sum_{k, \ell} q_{k, \ell} e^{i(k \alpha+\ell \beta) x}+o(1)$ when $x \rightarrow \infty$ for some incommensurable ${ }^{(\mathrm{i})}$ pair of real numbers $(\alpha, \beta)$ and complex numbers $\left\{q_{k, \ell}\right\}_{(k, \ell) \in \mathbb{Z}^{2}}$.

\subsubsection{The Irrational Case}

We now turn to the irrational case. Let $A>0$ be a number such that for all $|s| \leq A$ we have $\left|\lambda\left(c_{2}+s\right)\right|>$ $\left|\lambda_{2}\left(c_{2}+s\right)\right|$; thus by Lemma $9 L\left(c_{2}+s\right)$ is analytic. We assume that $c_{2}<0$ is the only saddle point on $\Re(s)=c_{2}$ for $|\Im(s)| \leq A$. We also postulate that there is $\alpha_{3}>0$ such that

$$
|t| \leq A \Rightarrow \Re\left(L\left(c_{2}+i t\right)-L\left(c_{2}\right)\right) \leq-\alpha_{3} t^{2} .
$$

From the previous analysis we know that

$$
\begin{gathered}
\frac{1}{2 i \pi} \sum_{k \in \mathbb{Z}} \int_{\Re(s)=c_{2},|\Im(s)| \leq A} \frac{f(s) g(s)}{\lambda(s) \log |\mathcal{A}|} \Xi\left(-L(s)-\frac{2 i k \pi}{\log |\mathcal{A}|}\right) \Xi(s) z^{L(s)-s+2 i k \pi / \log |\mathcal{A}|} d s= \\
=Q(\log z)(1+o(1)) .
\end{gathered}
$$

(i) A pair of numbers $(\alpha, \beta)$ is commensurable if there exists a real number $\nu$ such that the vector $(\nu \alpha, \nu \beta) \in \mathbb{Z}^{2}$; otherwise the pair is incommensurable. 
Assume now [38) and define

$$
\xi(s)=\sum_{k \in \mathbb{Z}}\left|\Xi\left(s-\frac{2 i k \pi}{\log |\mathcal{A}|}\right)\right| .
$$

The function $\xi(s)$ is continuous and bounded as long as $\Re(s)$ is bounded. Our aim is to prove that

$$
\frac{1}{2 i \pi} \int_{\Re(s)=c_{2},|\Im(s)|>A}\left|\frac{f(s) g(s)}{\lambda(s)}\right| \xi(-L(s))|\Xi(s)| z^{\Re(L(s))-c_{2}} d s=o\left(\frac{z^{\kappa}}{\sqrt{\log z}}\right),
$$

which completes the proof of Theorem 1

We know that $|f(s) g(s)| \leq f\left(c_{2}\right) g\left(c_{2}\right)$. In addition, we know that for $\Re(s)=c_{2}$ we have $\Re(L(s))<$ $L\left(c_{2}\right)$ as long as $\Im(s) \neq 0$. We also have $|\lambda(s)|>\epsilon^{\prime}$ for some $\epsilon^{\prime}>0$ since the matrix $\mathbf{P}(s)$ stays away from the null matrix. Therefore, we need to estimate

$$
\int_{\Re(s)=c_{2},|\Im(s)|>A}|\Xi(s)| z^{\Re(L(s))-c_{2}} d s .
$$

For any $\epsilon>0$, the portion of the line $\Re(s)=c_{2}$, where $\Re(L(s))<L\left(c_{2}\right)-\epsilon$, contributes $z^{\kappa-\epsilon}$ to $T(z, z)$. Our attention must turn to the values of $s$ on this line such that $\Re(L(s))$ is arbitrary close to $L\left(c_{2}\right)$. In particular, we are interested in the local maxima of $\Re(L(s))$ that are arbitrary close to $L\left(c_{2}\right)$. Indeed, these local maxima play a role in the saddle point method.

Let us consider the sequence of those maxima denoted by $s_{\ell}$ for $\ell \in \mathbb{N}$ such that $\Re\left(L\left(s_{\ell}\right)\right) \rightarrow L\left(c_{2}\right)$. By Lemma 9 we know that for all real $t L\left(s_{\ell}+i t\right)-L\left(s_{\ell}\right) \rightarrow L\left(c_{2}+i t\right)-L\left(c_{2}\right)$ and that $L^{\prime}\left(s_{\ell}+i t\right) \rightarrow$ $L^{\prime}\left(c_{2}+i t\right)$. Therefore for all real $t$ such $|t| \leq A$

$$
\lim _{\ell \rightarrow \infty}\left(\Re\left(L\left(s_{\ell}+i t\right)\right)-\Re\left(L\left(s_{\ell}\right)\right)\right) \leq-\alpha_{3} t^{2}
$$

We define $I(A)$ to be the set of complex numbers $s$ such that $\Re(s)=c_{2}$ and $\min _{\ell}\left\{\left|s-s_{\ell}\right|\right\}>A$.

Lemma 12 There exists $\epsilon$ such that for all $s \in I(A): \Re(L(s))<L\left(c_{2}\right)-\epsilon$.

Proof: Assume $s \in I(A)$. Since $s$ is not a local maxima, we study the variation of $\Re(L(s))$ around the local maxima $s_{\ell}$. Without loss of generality we assume that $s_{\ell}-A$ is between $s$ and $s_{\ell}$, thus $\Re\left(L\left(s_{\ell}-\right.\right.$ $A))>\Re(s)$. Since $\lim \sup \Re\left(L\left(s_{\ell}-A\right)\right)<L\left(c_{2}\right)-\alpha_{3} A^{2}<L\left(c_{2}\right)-\epsilon$ the lemma is proven.

In view of the above, we conclude that

$$
\int_{\Re(s)=c_{2},|\Im(s)|>A}|\Xi(s)| z^{\Re(L(s))-c_{2}} d s \leq \sum_{\ell} \int_{|t| \leq A}\left|\Xi\left(s_{\ell}+i t\right)\right| z^{\Re\left(L\left(s_{\ell}+i t\right)\right)-c_{2}} d t+O\left(z^{\kappa-\epsilon}\right) .
$$

Since $\Xi(s)=(s+1) \Gamma(s)$ on the line $\Re(s)=c_{2}$, there exists a real $B>0$ such that $\forall s$ :

$$
\Re(s)=c_{2} \Rightarrow \max _{|t| \leq A}\{|\Xi(s+i t)|\} \leq B|\Xi(s)| .
$$

Therefore, our analysis can be limited to

$$
\sum_{\ell} \int_{|t| \leq A}\left|\Xi\left(s_{\ell}\right)\right| z^{\Re\left(L\left(s_{\ell}+i t\right)\right)-c_{2}} d t
$$


Lemma 13 For $\ell$ tending to infinity, the $s_{\ell}$ are separated by a distance at least equal to $A$.

Proof: First, let us assume that $\ell, \ell^{\prime} \rightarrow \infty$ and $\left|s_{\ell}-s_{\ell^{\prime}}\right| \rightarrow 0$, then we have

$$
L^{\prime}\left(s_{\ell^{\prime}}\right)=L^{\prime}\left(s_{\ell}\right)+\left(s_{\ell^{\prime}}-s_{\ell}\right) L^{\prime \prime}\left(s_{\ell}\right)+O\left(\left|s_{\ell}-s_{\ell^{\prime}}\right|^{2}\right) .
$$

Since $L^{\prime \prime}\left(s_{\ell}\right) \rightarrow \alpha_{2} \neq 0$, then we cannot have $L^{\prime}\left(s_{\ell^{\prime}}\right)=1$, thus $s_{\ell^{\prime}}$ cannot be a local maximum of $\Re(L(s))$. Second, if liminf $\left|s_{\ell}-s_{\ell^{\prime}}\right|>\epsilon$ for some $\epsilon>0$ with $\left|s_{\ell}-s_{\ell^{\prime}}\right|<A$, then using the inequality

$$
\limsup \Re\left(L\left(s_{\ell^{\prime}}\right)\right)-\Re\left(s_{\ell}\right) \leq-\alpha_{3}\left|s_{\ell}-s_{\ell^{\prime}}\right|^{2}<-\alpha_{3} \epsilon^{2}
$$

we cannot have $\Re\left(L\left(s_{\ell^{\prime}}\right)\right) \rightarrow L\left(c_{2}\right)$.

The consequence of the previous lemma and the properties of function $\Xi(s)$ is that $\sum_{\ell}\left|\Xi\left(s_{\ell}\right)\right|<\infty$. Therefore,

$$
\sum_{\ell} \int_{|t| \leq A}\left|\Xi\left(s_{\ell}\right)\right| z^{\Re\left(L\left(s_{\ell}+i t\right)\right)-c_{2}} d t=z^{\kappa} \sum_{\ell}\left|\Xi\left(s_{\ell}\right)\right| z^{\Re\left(s_{\ell}\right)-L\left(c_{2}\right)} \int_{|t| \leq A} z^{\Re\left(L\left(s_{\ell}+i t\right)\right)-\Re\left(L\left(s_{\ell}\right)\right.} d t .
$$

Since $\lim \sup _{\ell \rightarrow \infty} \Re\left(L\left(s_{\ell}+i t\right)\right)-\Re\left(L\left(s_{\ell}\right) \leq-\alpha_{3} t^{2}\right.$, we have [13]

$$
\lim \sup _{\ell \rightarrow \infty} \int_{|t| \leq A} z^{\Re\left(L\left(s_{\ell}+i t\right)\right)-\Re\left(L\left(s_{\ell}\right)\right.} d t \leq \frac{1}{\sqrt{\pi \alpha_{3} \log z}},
$$

and since $\lim _{z \rightarrow \infty} z^{\Re\left(s_{\ell}\right)-L\left(c_{2}\right)}=0$, by the dominating convergence theorem, we arrive at

$$
\sum_{\ell}\left|\Xi\left(s_{\ell}\right)\right| z^{\Re\left(s_{\ell}\right)-L\left(c_{2}\right)} \int_{|t| \leq A} z^{\Re\left(L\left(s_{\ell}+i t\right)\right)-\Re\left(L\left(s_{\ell}\right)\right.} d t=o\left(\frac{1}{\sqrt{\log z}}\right) .
$$

The case $c_{2}>0$ is discussed in the Appendix. It relies the singularity method.

\subsection{Double Depoissonization}

To complete the proof of Theorem 1 we need to translate $T\left(z_{1}, z_{2}\right)$ into the original $T_{n, m}$. We accomplish it through a two-dimensional (double) depoissonization that we discuss next.

Let $a_{n, m}$ be a two-dimensional (double) sequence of complex numbers. We define the double Poisson transform $f\left(z_{1}, z_{2}\right)$ of $a_{n, m}$ as

$$
f\left(z_{1}, z_{2}\right)=\sum_{n, m \geq 0} \frac{z_{1}^{n}}{n !} \frac{z_{1}^{m}}{m !} e^{-z_{1}-z_{2}}
$$

It is relatively straightforward to extend the one-dimensional depoissonization result of [6] to the twodimensional case [4, 7]. In the Appendix we prove the following.

Lemma 14 Let $\mathcal{S}_{\theta}$ be a cone of angle $\theta$ around the real axis. Assume that there exist $B>0, D>0$, $\alpha<1$ and $\beta$ such that for $\left|z_{1}\right|,\left|z_{2}\right| \rightarrow \infty$ :

- if $z_{1}, z_{2} \in \mathcal{S}_{\theta}:\left|f\left(z_{1}, z_{2}\right)\right|=B\left(\left|z_{1}\right|^{\beta}+\left|z_{2}\right|^{\beta}\right)$; 
- if $z_{1}, z_{2} \notin \mathcal{S}_{\theta}:\left|f\left(z_{1}, z_{2}\right) e^{z_{1}+z_{2}}\right|=D e^{\alpha\left|z_{1}\right|+\alpha\left|z_{2}\right|}$;

- if $z_{i} \in \mathcal{S}_{\theta}$ and $z_{j} \notin \mathcal{S}_{\theta}$ for $\{i, j\}=\{1,2\}:\left|f\left(z_{1}, z_{2}\right) e^{z_{j}}\right|<D\left|z_{i}\right|^{\beta} e^{\alpha\left|z_{j}\right|}$.

Then

$$
a_{n, m}=f(n, m)+O\left(\frac{n^{\beta}}{m}+\frac{m^{\beta}}{n}\right) .
$$

This leads to our final result of this section.

Lemma 15 Generating function $T\left(z_{1}, z_{2}\right)$ satisfies the condition of Lemma 14 with $\beta=1$. Therefore,

$$
T_{n, m}=T(n, m)+O\left(\frac{n}{m}+\frac{m}{n}\right) .
$$

\section{References}

[1] P. Flajolet, X. Gourdon, and P. Dumas, Mellin Transforms and Asymptotics: Harmonic sums, Theoretical Computer Science, 144, 3-58, 1995.

[2] P. Flajolet and R. Sedgewick, Analytic Combinatorics, Cambridge University Press, Cambridge, 2008.

[3] Ilie, L., Yu, S., and Zhang, K. Repetition Complexity of Words In Proc. COCOON 320-329, 2002.

[4] P. Jacquet, Common words between two random strings, IEEE Intl. Symposium on Information Theory, 1495-1499, 2007.

[5] P. Jacquet, and W. Szpankowski, Autocorrelation on Words and Its Applications. Analysis of Suffix Trees by String-Ruler Approach, J. Combinatorial Theory Ser. A, 66, 237-269, 1994.

[6] P. Jacquet, and W. Szpankowski, Analytical Depoissonization and Its Applications, Theoretical Computer Science, 201, 1-62, 1998.

[7] P. Jacquet, and W. Szpankowski, Analytic Pattern Matching: DNA, Lempel-Ziv, and Trees, Cambridge Press, 2012.

[8] P. Jacquet, W. Szpankowski, and J. Tang, Average Profile of the Lempel-Ziv Parsing Scheme for a Markovian Source, Algorithmica, 31, 318-360, 2001.

[9] S. Janson, S. Lonardi and W. Szpankowski, On Average Sequence Complexity, Theoretical Computer Science, 326, 213-227, 2004.

[10] Li, M., and Vitanyi, P. Introduction to Kolmogorov Complexity and its Applications. Springer-Verlag, Berlin, Aug. 1993.

[11] Niederreiter, H., Some computable complexity measures for binary sequences, In Sequences and Their Applications, Eds. C. Ding, T. Hellseth and H. Niederreiter Springer Verlag, 67-78, 1999. 
[12] G. Park, H.K. Hwang, P. Nicodeme, and W. Szpankowski, Profile of Tries, SIAM J. Computing, 8, 1821-1880, 2009.

[13] W. Szpankowski, Analysis of Algorithms on Sequences, John Wiley, New York, 2001.

\section{Appendix}

\section{Proof of Lemma 1: We have}

$$
\begin{aligned}
\lambda(s) & =\langle\boldsymbol{\zeta}(s) \mid \mathbf{P}(s) \mathbf{u}(s)\rangle=\sum_{a, b} \zeta_{a}(s) u_{b}(s) P(a \mid b)^{-s}, \\
\lambda^{\prime}(s) & =\left\langle\boldsymbol{\zeta}(s) \mid \mathbf{P}^{\prime}(s) \mathbf{u}(s)\right\rangle=\sum_{a, b} \zeta_{a}(s) u_{b}(s) P(a \mid b)^{-s}(-\log P(a \mid b)), \\
\lambda^{\prime \prime}(s) & =\left\langle\boldsymbol{\zeta}(s) \mid \mathbf{P}^{\prime \prime}(s) \mathbf{u}(s)\right\rangle=\sum_{a, b} \zeta_{a}(s) u_{b}(s) P(a \mid b)^{-s}(\log P(a \mid b))^{2} .
\end{aligned}
$$

Let $x_{a, b}=\frac{1}{\lambda(s)} \zeta_{a}(s) u_{b}(s) P(a \mid b)^{-s}$, we have $\sum_{a, b} x_{a, b}=1$. Also

$$
L^{\prime \prime}(s)=\sum_{a, b} x_{a, b}(\log P(a \mid b))^{2}-\left(\sum_{a, b} x_{a, b} \log P(a \mid b)\right)^{2} .
$$

By the Perron Frobenius theorem $\boldsymbol{\zeta}(s)$ and $\mathbf{u}(s)$ have positive coefficients. Hence, by the convexity of the quadratic function we must have $L^{\prime \prime}(s) \geq 0$.

Proof of Lemma 2; For $c_{2}>-1$ it suffices to show $L^{\prime}(-1)<1$. Since $\lambda(-1)=1$, we we only need to prove that $\lambda^{\prime}(-1)<\log |\mathcal{A}|$. We also have $\zeta(-1)=1$. Observe

$$
\lambda^{\prime}(-1)=\sum_{a, b} \zeta_{a}(-1) u_{b}(-1)(-P(a \mid b) \log P(a \mid b)) .
$$

Since $\sum_{a, b} \zeta_{a}(-1) u_{b}(-1)=\langle\boldsymbol{\zeta}(-1) \mid \mathbf{1}\rangle\langle\mathbf{1} \mid \mathbf{u}(-1)\rangle=|\mathcal{A}|$, and by concavity of the function $-x \log x$ on the interval $[0,1]$ we have

$$
\lambda^{\prime}(-1)<-\left(\sum_{a, b} \zeta_{a}(-1) u_{b}(-1) P(a \mid b)\right) \log \left(\sum_{a, b} \frac{1}{|\mathcal{A}|} \zeta_{a}(-1) u_{b}(-1) P(a \mid b)\right)=\log |\mathcal{A}| ;
$$

the inequality is strict because the $P(a \mid b)$ 's are not all identical.

For $c_{2}<0$, when all $P(a \mid b)>0$, it suffices to prove that $L^{\prime}(0)>1$. Then by the increasing nature of $L^{\prime}(s)$ we will necessarily have $c_{2}<0$. We have $\mathbf{P}(0)$ entirely made of ones, thus $\lambda(0)=|\mathcal{A}|$ and $\mathbf{u}(0)=\frac{1}{|\mathcal{A}|} \mathbf{1}$ and $\boldsymbol{\zeta}(0)=\mathbf{1}$. By convexity of $-\log$ function, and since the $P(a \mid b)$ 's are not all identical

$$
\frac{\lambda^{\prime}(0)}{\lambda(0)}=-\sum_{a, b} \frac{1}{|\mathcal{A}|^{2}} \log P(a \mid b)>-\log \left(\sum_{a \mid b} \frac{1}{|\mathcal{A}|^{2}} P(a \mid b)\right) .
$$

Since $\sum_{a, b} P(a \mid b)=|\mathcal{A}|$ we get $\frac{\lambda^{\prime}(0)}{\lambda(0)}>\log |\mathcal{A}|$. 
Proof of Lemma 4; We move the integration line in $I(z, \rho)$ from the vertical line $\Re\left(s_{2}\right)=\rho$ to the vertical line $\Re\left(s_{2}\right)=c_{2}$. The function

$$
J_{k}(s, z)=\sum_{i} \frac{f_{i}(s) g_{i}(s)}{\lambda_{i}(s)} \Xi\left(-L_{i, k}(s)\right) z^{L_{i, k}(s)}
$$

satisfies the (somewhat complicated) identity

$J_{k}(s, z)=\left\langle\boldsymbol{\pi}(s) \mid(\mathbf{P}(s))^{-1} \exp \left(-\frac{\log z}{\log |\mathcal{A}|}(\log \mathbf{P}(s)+2 i k \pi \mathbf{I})\right) \Xi\left(-\frac{1}{\log |\mathcal{A}|}(\log \mathbf{P}(s)+2 i k \pi \mathbf{I})\right) \mathbf{1}\right\rangle$,

knowing that any analytical function $f$ can be applied to matrix $\mathbf{P}(s)$ as long its eigenvalues do not correspond to a singularity of $f$. Therefore the only singularities that we meet when we move the integration line of $I(z, \rho)$ are the elements of $\mathcal{R}=\left\{s: \lambda_{i}(s)=0\right.$, for some $\left.i\right\}$.

We have

$$
I(z, \rho)=\frac{1}{2 i \pi} \int_{\Re(s)=\rho} \sum_{i} H_{i}(s, z) \Xi(s) z^{-s} d s .
$$

If $\theta \in \mathcal{R}$, and we have $\lambda_{i}(\theta)=0$, then the function $L_{i, k}(s)=\frac{1}{-\log |\mathcal{A}|}\left(\log \lambda_{i}(s)+2 i k \pi\right)$ is meromorphic around $\theta$. However if $\theta$ is a simple root of $\lambda_{i}(s)$, then moving around $\theta$ would be equivalent to add 1 to the integer $k: \log \lambda_{i}(s) \rightarrow \log \lambda_{i}(s)+2 i \pi$. If the root if of multiplicity $\ell$ it is equivalent to add $\ell$ to the integer $k$. In any case the function $H_{i}(s, z)$ being invariant when $\ell$ is added to $k$, turns out to be fully analytic around $\theta$, and the integration path in $I(z, \rho)$ can be moved over $\theta$.

However, the function $H_{i}(s, z)$ is still singular on $s=\theta$, hence there will be a contribution coming from the integration of $H_{i}(s, z) \Xi(s) z^{-s}$ on an arbitrary small loop around $\theta$. Since $\Re\left(L_{i, k}(s)\right) \rightarrow-\infty$ when $s \rightarrow \theta$, having $\Re\left(L_{i, k}(s)\right)<-M$ will guarantee that the contribution is in $O\left(z^{1-M}\right)$ and can be included in the error term.

Proof of Lemma 5 Let $\mathbf{u}=\left(u_{a}\right)_{a \in \mathcal{A}}$ be the right eigenvector of $\mathbf{M}$ and $\left(v_{a}\right)_{a \in \mathcal{A}}$ be the right eigenvector of $\mathbf{Q}$. Let also $v_{a}=x_{a} u_{a}$. If 1 is the eigenvalue, we have for all $c \in \mathcal{A}$ :

$$
\left(1-e^{i \theta_{c c}} m_{c c}\right) u_{c}=\sum_{b \neq c} m_{c b} u_{b} e^{i \theta_{c b}} \frac{x_{b}}{x_{c}} .
$$

If $e^{i \theta_{c c}} \neq 1$, then

$$
\left|\left(1-e^{i \theta_{c c}} m_{c c}\right) u_{c}\right|>\left(1-m_{c c}\right) u_{c} .
$$

By the Perron-Frobenius theorem all $u_{a}$ are real non negative. Suppose that $\left|x_{c}\right|=\max _{a \in \mathcal{A}}\left\{\left|x_{a}\right|\right\}$. If $\exists d \in \mathcal{A}: \frac{\left|x_{d}\right|}{\left|x_{c}\right|}<1$ or if $\left(b, b^{\prime}\right) \in(\mathcal{A}-\{c\})^{2}: e^{i \theta_{c b}} \frac{x_{b}}{x_{c}} \neq e^{i \theta_{c b^{\prime}}} \frac{x_{b}^{\prime}}{x_{c}}$. Then

$$
\left|\sum_{b \neq c} m_{c b} u_{b} e^{i \theta_{c b}} \frac{x_{b}}{x_{c}}\right|<\sum_{b \neq c} m_{c b} u_{b} .
$$

But we also know that

$$
\left(1-m_{c c}\right) u_{c}=\sum_{b \neq c} m_{c b} u_{b}
$$


Therefore, we have $e^{i \theta_{c c}}=1$ and for all $b \in \mathcal{A}:\left|x_{b}\right|=\left|x_{c}\right|$, and for all $\left(b, b^{\prime}\right) \in(\mathcal{A}-\{c\})^{2}: e^{i \theta_{c b}} \frac{x_{b}}{x_{c}}=$ $e^{i \theta_{c b^{\prime}}} \frac{x_{b}^{\prime}}{x_{c}}$. But since for all $b \in \mathcal{A}\left|x_{b}\right|=\left|x_{c}\right|$ every symbol in $\mathcal{A}$ can play the role of $c$. Since for all $c \in \mathcal{A}$

$$
\left(1-m_{c c}\right)=\sum_{b \neq c} m_{c b} u_{b} e^{i \theta_{c b}} \frac{x_{b}}{x_{c}}=\sum_{b \neq c} m_{c b} u_{b}
$$

we simply have $\forall(a, b) \in \mathcal{A}: e^{i \theta_{a b}} \frac{x_{b}}{x_{a}}=1$. Denoting $x_{a}=e^{i \theta_{a}}$ we get the expected result. The inverse proposition is immediate.

Proof of Lemma 9: Consider the matrix $\frac{1}{\lambda\left(s_{k}\right)} \mathbf{P}\left(s_{k}\right)$. Since the coefficients of this matrix are bounded, there is no loss in generality to consider the sequence of matrices converging to a matrix $\mathbf{M}$. The matrix $\mathbf{M}$ and matrix $\mathbf{Q}=\frac{1}{\lambda\left(c_{2}\right)} \mathbf{P}\left(c_{2}\right)$ defined in Lemma 5 are imaginary conjugate i.e. the coefficients of $\mathbf{M}$ are of the form

$$
e^{i\left(\theta_{a}-\theta_{b}\right)} \frac{1}{\lambda\left(c_{2}\right)} P(a \mid b)^{-c_{2}}
$$

for some vector of real numbers $\theta_{a}$. Therefore, $\mathbf{M}$ and $\frac{1}{\lambda\left(c_{2}\right)} \mathbf{P}\left(c_{2}\right)$ have the same spectrum. The spectrum of $\frac{1}{\lambda\left(s_{k}\right)} \mathbf{P}\left(s_{k}\right)$ converges to the spectrum of $\mathbf{M}$. Furthermore, the right eigenvector $\mathbf{u}\left(s_{k}\right)$ converges to the vector $e^{i \theta_{a}} u_{a}\left(c_{2}\right)$ and the left eigenvector $\zeta\left(s_{k}\right)$ converges to $e^{-i \theta_{a}} \zeta_{a}\left(c_{2}\right)$.

For any complex number $s$ we have the identity

$$
\frac{1}{\lambda\left(s_{k}\right)} \mathbf{P}\left(s_{k}+s\right)=\frac{1}{\lambda\left(s_{k}\right)} \mathbf{P}\left(s_{k}\right) * \mathbf{P}(s) .
$$

Thus $\frac{1}{\lambda\left(s_{k}\right)} \mathbf{P}\left(s_{k}+s\right)$ converges to $\mathbf{M} * \mathbf{P}(s)$ and is conjugate to $\frac{1}{\lambda\left(c_{2}\right)} \mathbf{P}\left(c_{2}+s\right)$. Hence, the logarithm of the eigenvalue $L\left(s_{k}+s\right)-L\left(s_{k}\right)$ converges to $L\left(c_{2}+s\right)-L\left(c_{2}\right)$. The property $\left|\lambda\left(c_{2}+s\right)\right|>\lambda_{2}\left(c_{2}+s\right)$ for all $s \in \mathcal{U}$ implies the analyticity of $L\left(c_{2}+s\right)$, and therefore $L^{\prime}\left(c_{2}+s\right)$. Since the eigen spectrum of $\frac{1}{\lambda\left(s_{k}\right)} \mathbf{P}\left(s_{k}+s\right)$ converges to the eigen spectrum of $\frac{1}{\lambda\left(c_{2}\right)} \mathbf{P}\left(c_{2}+s\right)$, we also have $\lambda\left(s_{k}+s\right)>\lambda_{2}\left(s_{k}+s\right)$ when $k$ is large enough which implies the analyticity of $L\left(s_{k}+s\right)$. Thus by Ascoli theorem the derivatives converge, too.

Proof of Theorem 1 for the case $c_{2}>0$ : While moving the integration path from $\Re(s)=\rho$ to $\Re(s)=c_{2}$, as in Lemma 4 we meet the pole of $\Xi(s)$ at $s=0$. There, the function $\sum_{i} H_{i}(s, n) \Xi(s) n^{-s}$ has residue $\sum_{i} H_{i}(0, n)$. Thus for all $M>0$ :

$$
T(n, n)=\sum_{i} H_{i}(0, n)+I\left(c_{2}, n\right)+O\left(n^{1-M}\right) .
$$

We notice that $I\left(c_{2}, n\right)=O\left(n^{\kappa}\right)$ which is $O\left(n^{L(0)-\epsilon}\right)$. Furthermore,

$$
\sum_{i} H_{i}(0, n)=\sum_{i} \frac{f_{i}(0) g_{i}(0)}{\lambda_{i}(0)} \sum_{k \in \mathbb{Z}} \Xi\left(-L_{i}(s)-\frac{2 i k \pi}{\log |\mathcal{A}|}\right) n^{L_{i}(0)+2 i k \pi / \log |\mathcal{A}|},
$$

and for $i>1, L_{i}(0)<L(0)-\epsilon$. Thus $T(n, n)=H_{1}(0, n)+O\left(n^{L(0)-\epsilon}\right)$. 
Proof of Lemma 14: Let

$$
f_{n}\left(z_{2}\right)=\sum_{m} a_{n, m} \frac{z_{2}^{m}}{m !} e^{-z_{2}} .
$$

We notice that $f\left(z_{1}, z_{2}\right)$ is the Poisson transform of the sequence $f_{n}\left(z_{2}\right)$ with respect to the variable $z_{1}$. First depoissonization. For $z_{2} \in \mathcal{S}_{\theta}$ we have the estimates

$$
\begin{aligned}
z_{1} \in \mathcal{S}_{\theta}:\left|f\left(z_{1}, z_{2}\right)\right| & <B\left(\left|z_{1}\right|^{\beta}+\left|z_{2}\right|^{\beta}\right) \\
z_{1} \notin \mathcal{S}_{\theta}:\left|f\left(z_{1}, z_{2}\right) e^{z_{1}}\right| & <D\left|z_{2}\right|^{\beta} e^{\alpha\left|z_{1}\right|} .
\end{aligned}
$$

Therefore, from the one-dimensional analytic depoissonization of [6, 13] for $z_{2} \in \mathcal{S}_{\theta}$, we have for all integers $k>0$

$$
f_{n}\left(z_{2}\right)=f\left(n, z_{2}\right)+O\left(n^{\beta-1}+\frac{\left|z_{2}\right|^{\beta}}{n}\right)+O\left(\left|z_{2}\right|^{\beta} n^{\beta-k}\right) .
$$

Similarly, when $z_{2} \notin \mathcal{S}_{\theta}$ we have

$$
\begin{aligned}
z_{1} \in \mathcal{S}_{\theta}:\left|f\left(z_{1}, z_{2}\right) e^{z_{2}}\right| & <D\left|z_{1}\right|^{\beta} e^{\alpha\left|z_{2}\right|} \\
z_{1} \notin \mathcal{S}_{\theta}:\left|f\left(z_{1}, z_{2}\right) e^{z_{1}+z_{2}}\right| & <D e^{\alpha\left|z_{1}\right|+\alpha\left|z_{2}\right|} .
\end{aligned}
$$

Thus for all integer $k$ and $\forall z_{2} \notin \mathcal{S}_{t} h$

$$
f_{n}\left(z_{2}\right) e^{z_{2}}=f\left(n, z_{2}\right) e^{z_{2}}+O\left(n^{\beta-1} e^{\alpha\left|z_{2}\right|}\right)+O\left(n^{\beta-k} e^{\alpha\left|z_{2}\right|}\right) .
$$

Second depoissonization The two results on $f_{n}\left(z_{2}\right)$, respectively for $z_{2} \in \mathcal{S}_{\theta}$ and $z_{2} \notin \mathcal{S}_{\theta}$, allow us to depoissonize $f_{n}\left(z_{2}\right)$. For all $k>\beta$ :

- for $z_{2} \in \mathcal{S}_{\theta}: f_{n}\left(z_{2}\right)=O\left(n^{\beta}+\left|z_{2}\right|^{\beta}\right)$;

- for $z_{2} \notin \mathcal{S}_{\theta}: f_{n}\left(z_{2}\right) e^{z_{2}}=O\left(n^{\beta} e^{\alpha\left|z_{2}\right|}\right)$.

These estimates are uniform. Therefore,

$$
a_{n, m}=f_{n}(m)+O\left(\frac{n^{\beta}}{m}+\frac{m^{\beta}}{n}\right)+O\left(n^{\beta} m^{\beta-k}\right) .
$$

Since

$$
f_{n}(m)=f(n, m)+O\left(n^{\beta-1}+\frac{m^{\beta}}{n}\right)
$$

and setting $k>\beta+1$, we prove the desired estimate.

Proof of Lemma 15: To prove the lemma, we need to establish three conditions (i)-(iii) of Lemma 14. We accomplish it through a generalization of the so called increasing domain approach discussed in [6, 13].

We first prove the lemma for the generating functions $T_{a}\left(z_{1}, z_{2}\right)$ for every $a \in \mathcal{A}$. Assume now that $r=\max _{(a, b) \in \mathcal{A}^{2}, i \in\{1,2\}}\left\{P_{i}(a \mid b)\right\}$. We denote $\mathcal{S}_{k}$ part of the cone $\mathcal{S}_{\theta}$ that contains points such that $|z|<\rho^{-k}$. Notice that $\mathcal{S}_{k} \subset \mathcal{S}_{k+1}$ for all integer $k$. We also notice $C\left(z_{1}, z_{2}\right)=O\left(\left(\left|z_{1}\right|+\left|z_{2}\right|\right)^{2}\right)$ when $z_{1}, z_{2} \rightarrow 0$, therefore we can define

$$
B_{k}=\max _{a \in \mathcal{A},\left(z_{1}, z_{2}\right) \in \mathcal{S}_{k} \times \mathcal{S}_{k}} \frac{\left|T_{a}\left(z_{1}, z_{2}\right)\right|}{\left|z_{1}\right|+\left|z_{2}\right|}<\infty .
$$


We use the functional equation $[3$ :

$$
T_{b}\left(z_{1}, z_{2}\right)=\left(1-\left(1+z_{1}\right) e^{-z_{1}}\right)\left(1-\left(1+z_{2}\right) e^{-z_{2}}\right)+\sum_{a \in \mathcal{A}} T_{a}\left(P_{1}(a \mid b) z_{1}, P_{2}(a \mid b) z_{2}\right)
$$

In the above equation, we notice that if $\left(z_{1}, z_{2}\right) \in \mathcal{S}_{k+1} \times \mathcal{S}_{k+1}-\mathcal{S}_{k} \times \mathcal{S}_{k}$, then for all $(a, b) \in \mathcal{A}^{2}$ $\left(P_{1}(a \mid b) z_{1}, P_{2}(a \mid b) z_{2}\right)$ are in $\mathcal{S}_{k} \times \mathcal{S}_{k}$ and therefore we have for some fixed $\beta>0$ and for all $b \in \mathcal{A}$ :

$$
\left|T_{b}\left(z_{1}, z_{2}\right)\right| \leq B_{k}\left(\sum_{a \in \mathcal{A}} P_{1}(a \mid b)\left|z_{1}\right|+P_{2}(a \mid b)\left|z_{2}\right|\right)+\beta=B_{k}\left(\left|z_{1}\right|+\left|z_{2}\right|\right)+\beta
$$

since $\left|1-\left(1+z_{i}\right) e^{-z_{i}}\right|$ is uniformly bounded for all integers $k$ by some $\sqrt{\beta}$ for both $i \in\{1,2\}$ when $\left(z_{1}, z_{2}\right) \in \mathcal{S}_{k}$. Thus, we can derive the following recurrent inequality:

$$
B_{k+1} \leq B_{k}+\beta \max _{\left(z_{1}, z_{2}\right) \in \mathcal{S}_{k+1} \times \mathcal{S}_{k+1}-\mathcal{S}_{k} \times \mathcal{S}_{k}}\left\{\frac{1}{\left|z_{1}\right|+\left|z_{2}\right|}\right\}=B_{k}+\beta \rho^{k} .
$$

We should notice that

$$
\min _{\left(z_{1}, z_{2}\right) \in \mathcal{S}_{k+1} \times \mathcal{S}_{k+1}-\mathcal{S}_{k} \times \mathcal{S}_{k}}\left\{\left|z_{1}\right|+\left|z_{2}\right|\right\}=\rho^{-k}
$$

because maybe only one of the number $z_{i}$ has modulus greater than $\rho^{-k}$. It turns out that $\lim _{k \rightarrow \infty} B_{k}<$ $\infty$, establishing condition (i) of Lemma 14

Now we are going to establish condition (iii). For this end we define $\mathcal{G}$ as the complementary cone of $\mathcal{S}_{\theta}$ and $\mathcal{G}_{k}$ as the portion made of the point of modulus smaller than $\rho^{-k}$. We will use $\cos \theta<\alpha<1$, therefore $\forall z \in \mathcal{G}$ : $\left|e^{z}\right|<e^{\alpha|z|}$. We define $D_{k}$ as

$$
D_{k}=\max _{a \in \mathcal{A},\left(z_{1}, z_{2}\right) \in \mathcal{G}_{k} \times \mathcal{G}_{k}} \frac{\left|T_{a}\left(z_{1}, z_{2}\right) e^{z_{1}+z_{2}}\right|}{\exp \left(\alpha\left|z_{1}\right|+\alpha\left|z_{2}\right|\right)}
$$

We define $G_{a}\left(z_{1}, z_{2}\right)=T_{a}\left(z_{1}, z_{2}\right) e^{z_{1}+z_{2}}$, we have the equation

$G_{b}\left(z_{1}, z_{2}\right)=\left(e^{z_{1}}-1-z_{1}\right)\left(e^{z_{2}}-1-z_{2}\right)+\sum_{a \in \mathcal{A}} T_{a}\left(P_{1}(a \mid b) z_{1}, P_{2}(a \mid b) z_{2}\right) e^{1-P_{1}(a \mid b) z_{1}+\left(1-P_{2}(a \mid b)\right) z_{2}}$

We notice that if $\left(z_{1}, z_{2}\right) \in \mathcal{G}_{k+1} \times \mathcal{G}_{k+1}-\mathcal{G}_{k} \times \mathcal{G}_{k}$, then all $\left(P_{1}(a \mid b) z_{1}, P_{2}(a \mid b) z_{2}\right)$ are in $\mathcal{G}_{k} \times \mathcal{G}_{k}$ and therefore we have for all $b \in \mathcal{A}$ :

$$
\begin{aligned}
\left|G_{b}\left(z_{1}, z_{2}\right)\right| \leq & D_{k}\left(\sum_{a \in \mathcal{A}} \exp \left(\left(P_{1}(a \mid b) \alpha+\left(1-P_{1}(a \mid b)\right) \cos \theta\right)\left|z_{1}\right|+\left(P_{2}(a \mid b) \alpha+\left(1-P_{2}(a \mid b)\right) \cos \theta\right)\left|z_{2}\right|\right)\right) \\
& +\left(e^{\cos \theta\left|z_{1}\right|}+1+\left|z_{1}\right|\right)\left(e^{\cos \theta\left|z_{2}\right|}+1+\left|z_{2}\right|\right) .
\end{aligned}
$$

We notice that $\forall(a, b) \in \mathcal{A}^{2}$ and $\forall i \in\{1,2\}$ :

$$
P_{i}(a \mid b) \alpha+\left(1-P_{i}(a \mid b)\right) \cos \theta-\alpha \leq-(1-\rho)(\alpha-\cos \theta),
$$


We also have $e^{\cos \theta\left|z_{i}\right|}+1+\left|z_{i}\right| \leq e^{\cos \theta\left|z_{i}\right|}\left(2+\frac{1}{e \cos \theta}\right)$, therefore

$$
\frac{\left|G_{b}\left(z_{1}, z_{2}\right)\right|}{\exp \left(\alpha\left(\left|z_{1}\right|+\left|z_{2}\right|\right)\right)} \leq D_{k}|\mathcal{A}| e^{-(1-\rho)(\alpha-\cos \theta)\left(\left|z_{1}\right|+\left|z_{2}\right|\right)}+\left(2+\frac{1}{e \cos \theta}\right)^{2} e^{-(\alpha-\cos \theta)\left(\left|z_{1}\right|+\left|z_{2}\right|\right)} .
$$

Since $\left(z_{1}, z_{2}\right) \in \mathcal{G}_{k+1} \times \mathcal{G}_{k+1}-\mathcal{G}_{k} \times \mathcal{G}_{k}$ implies $\left|z_{1}\right|+\left|z_{2}\right| \geq \rho^{-k}$ it follows

$$
D_{k+1} \leq \max \left\{D_{k},|\mathcal{A}| D_{k} e^{-(1-\rho)(\alpha-\cos \theta) \rho^{-k}}+\left(2+\frac{1}{e \cos \theta}\right)^{2} e^{-(\alpha-\cos \theta) \rho^{-k}}\right\} .
$$

We clearly have $\lim _{k \rightarrow \infty} D_{k}<\infty$ and condition (iii) is established.

The proof of condition (ii) for $z_{1}$ and $z_{2}$ being in $\mathcal{S}_{\theta}$ and $\mathcal{G}$ is a mixture of the above proofs. Furthermore, the proof about the unconditional generating function $T\left(z_{1}, z_{2}\right)$ is a trivial extension of the one in (5). 